\title{
Electrocutions - Therapeutic Management - Results
}

\author{
${ }^{1}$ PhD. of Faculty of Medicine, "Ovidius" University of Constanta \\ ${ }^{2}$ Faculty of Medicine, "Ovidius" University of Constanta, Discipline- Anatomy \\ ${ }^{3}$ Faculty of Pharmacy, "Ovidius" University of Constanta \\ ${ }^{4}$ Faculty of Medicine, "Ovidius" University of Constanta, Discipline- Plastic Surgery;
}

\begin{abstract}
The authors present a retrospective study over a period of 18 years on electrocutions that were included electrocutions through direct contact (as the clinical presentation) or that were produced by high voltage or low voltage, which aims at extracting conclusions prognostic and therapeutic nature and especially the final therapeutic results, the study concluded that even electrocutions is decreases incidence, mortality of electrocutions with high voltage remains strong.
\end{abstract}

Keywords:Electrical injuries,high/low voltage

Iordache I. V.

Romania, Constanta,

Street: The Boulevard 1st December1918, Number:37, Block of flats: L21, Staircase: A, Apartment number: 15,

email: iordacheionutvalentin@gmail.com, phone: +40721000052

\section{Historic}

Injuries caused by electric current on the human body have been reported in about 300 years ago and the first death was reported in 1879 that in 1890 William Lyon Kemmeler to be the first man sentenced to death in the electric chair in New York [1].

The rate and severity of injuries caused by electric current on the human body have increased in parallel with the increasing use of electricity in industry and in the domestic environment especially where individual protective measures (labor protection) have been neglected. 


\section{Introduction}

Electrocutions represents a particular type of trauma, but the reasons clinical course and prognosis, is divided into: electrocutions with high voltage and low voltage electrocutions.

Electrocutions exact pathophysiology is not fully understood given the number of variables that can not be quantified or controlled passage of electric current. If electrocutions with high voltage produced varying degrees of burns associated with destruction hidden deep and wide; most injuries are thermal and histopathological studies revealed necrotic coagulation [2].

The main lesion directly is burning but appear related injuries - indirect - that are more evident when the victim becomes part of the arc and when developing $2500^{\circ} \mathrm{C}$ and injuries occur extremely deep in association further damage induced destructions vascular (thrombosis, damage electric vessels) with extensive secondary necrosis.

In terms of clinical and therapeutic use are described four types of lesions:

- electrocutions by direct contact; direct current passes through the body causing burns electrothermal;

- voltaic arch: is the electric current discharge between two points representing a potential difference without actually passing through the human body (the electrical circuit is closed due to ionization);

- flame burns: produces injuries by electrothermal effect but also burns cause by ignition flame of clothes (clothing);

- Flash type burns: thermal burns are produced by the energy released by an electric arc without electrical current to cross body.

There susceptibility to electrocutions related to sex, but it is estimated that approx. $80 \%$ of patients are men electrocuted [3].

Patients electrocuted - specifically the highvoltage electrocutions - shows serious risk of renal failure and secondary to myoglobinuria [4].

Therapeutic essential element in major electrocutions is represented by thesurgical treatment [5] and consisted of: debridement, pressure relief (by fasciotomy and fasciectomy), repeated excision of extending necrosis and coverage (as rapidly as possible) with autologus free skin graft (or substitutes leather) with the purpose of conservation of noble vital structures (blood vessels, nerves, muscles).

It can be said without mistake, in that the electrocution is not a disease but a syndrome and the treatment is that of a serious polytrauma patient [6].

\section{Material and method}

After over 37 years of experience in caring for patients with burns and electrocutions we considered it appropriate to conduct a retrospective study over a period of 18 years (1997-2014) on electrocutions in which we included electrocutions through direct contact (as the clinical presentation) or that were produced by high voltage or low voltage.

The study is targeted extracting some conclusions of therapeutic and prognostic and therapeutic outcomes specifically finals.

It should be stressed that the service of plastic surgery and burn in Constanta County Hospital cares for patients (acute and chronic) ascribed a territory with a total of about 500.000 inhabitants and that all electrocutions (at least initially) at hospital.

Throughout the study period retrospective clinical follow a mandatory protocol for treating all electrocutions and especially those with direct contact given that a number of electrocutions pass - at least after the initial stage of diagnosis and treatment - the application specific protocol burns.

The therapeutic plan envisages that the electrocuted is always a big shocked patient, dehydrated and potentially infected [7].

Treatment of a electrocuted represents a major emergency but also a corresponding qualification of the personnel since the release of the hotbed and to the post surgicalkinetotherapeutic treatment [6].

The measures of cardiac resuscitation is consistently applied whenever necessary, and surgical intervention is performed without delay, and consists 
of necrectomy wide (and repeated) as the realization of vascular release (fasciotomy, fasciectomy) and if required amputation the blunt will never be closed primary (per primam) as it leaves open the wound and after necrectomy.

After necrectomy is open surgical path of the electrical current traveled up where there was no possibility of achieving compression of large blood vessels even if at the surface are tissues of normal aspect (typically 5 to $12 \mathrm{~cm}$ from the edge of necrosis).

Is acclaimed cardiologist heart condition (clinical and ECG) since admission and whenever the situation requires.

General treatment goals are: to prevent and / or combat shock; avoiding secondary renal insufficiency and avoiding toxic-septic shock; which is done by: correcting electrolyte disorders, intense and sustained infusion, anticoagulants, antiaggregants, vasodilators, antiseptic, broad spectrum antibiotics, antienzymatics and painkillers.

All patients included in batch have benefited from the therapeutic protocol described above with updates about the drugs used in the treatment of general and local therapeutic procedures.

The age of the all included in batch patients is comprised between 16- 68 years of both sexes and were not included in the batch patients who underwent clinical forms electrocutions other than directly contact electrocutions.

High-voltage electrocutionsoccurred at tension of 10,000 or 27,000 volts and the low voltage electrocutions at 220 or 380 volts.

\section{Results - Discussions}

Distribution per year of the electrocutions with high and low voltage (Figure 1):

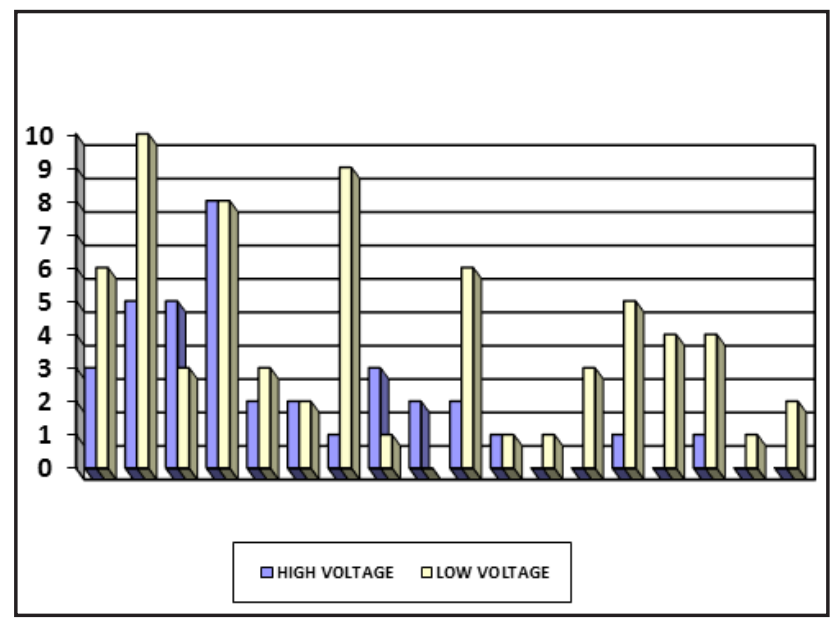

Figure 1 Distribution per year of the electrocutions with high and low voltage

As you can see above the number of electrocutions especially high voltage ones electrocutions have a significant decrease in number.

As for the gender distributionwe obtained a percentage a mere $2 \%$ women than $98 \%$ men.

And in all cases involving women, they suffered low voltage electrocutions.

In the comparationbetween deceased cases on high voltage per low voltage we can observe that all cases whith deceased patients was involved high voltage electrocutions.

General mortality on high voltage electrocutions we exposed schematically below. (Figure 2)

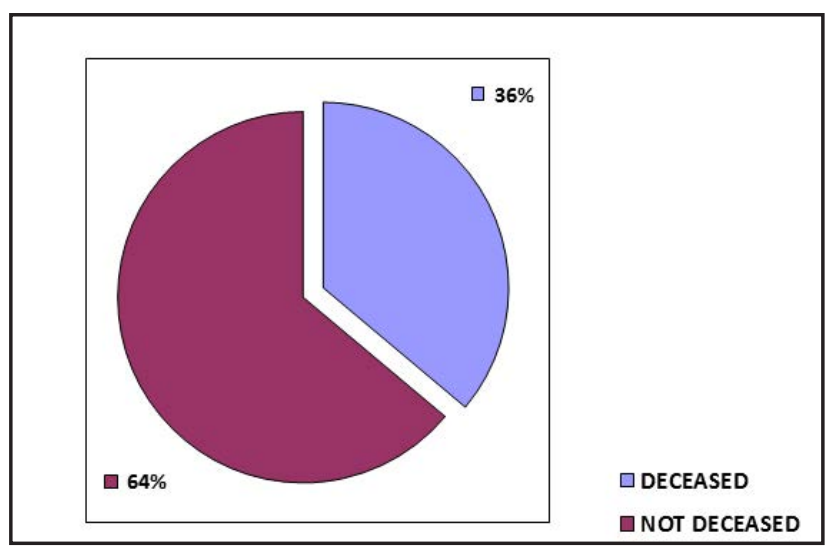

Figure 2 - General mortality on high voltage electrocutions

General mortality on low voltage electrocutions 
was zero.

Average length of hospitalization on low voltage electrocutions was 15 days 9 hours and 24 minutes, on the other hand average length of hospitalization on high voltage electrocutions was 47 days and 10 hours.(Figure 3)

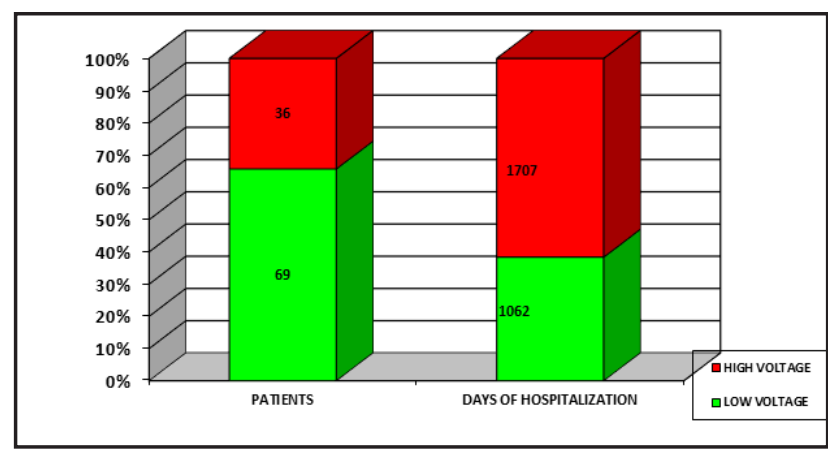

Figure 3

Average length of hospitalization on all electrocutions cases was 26 days 8 hours and 54 minutes.

The percentage of cases in which the required amputations (Figure 4)

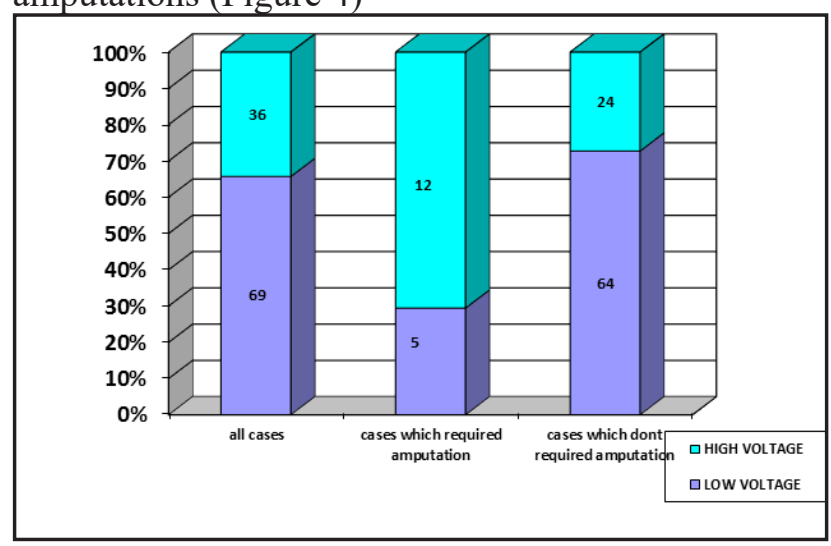

Figure 4

Average age of all cases was 34 years and 5 months.(Figure 5)

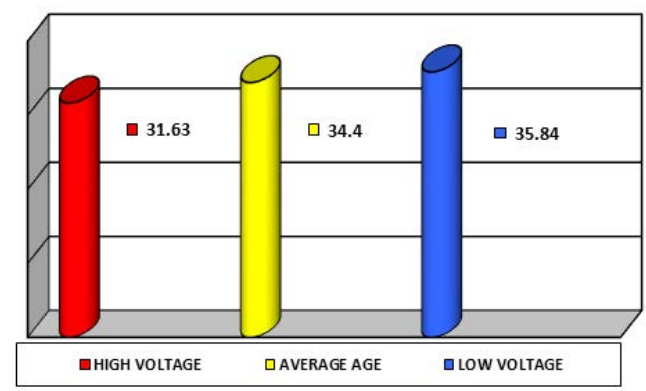

Figure 5

\section{Conclusions}

Electrocutions (especially those with highvoltage) is one of the most serious traumatic injuries to that the human body can be subjected and one of the highest risk invalidating traumatic injuries.

Even if there is a sex-linked susceptibility to the electrocuting them, in our study electrocutions occurred in $98 \%$ in men.

Patients with electrocutions a large proportion are young - the average age in our study was 34 years and 5 months.

High-voltage electrocutions mortality is $36 \%$ and remained high despite the consistent implementation of all current therapeutic procedures (both in the local treatment and in general).

From our study ensue that in recent years (from those included in the study) electrocuions incidence is clearly declining as a result of preventive measures.

\section{References}

1. Kobernic M. (1982). Electrical injuries, Pathophysiology, Manifestations and 
Management, CunEmerg. Med; 11(11),633-638.

2. Jain S. \& Bandi V. (1999). Electrical Lightening Injuries, Chir. Care Clin., 15(2), 319-31.

3. Luz D.P., Millam P.S., Alessis M.S., Uguetto W.F., Paggiaro A., Gomez D.S. \& Ferreira M.C. (2009). Electrical burns: a retrospective analysis across a 5-year period; Burns, 35(7), 1015-9.

4. Koop J., Loos B., Spilher G. \& Horch R.E. (2004). Correlation between serum creatinine kinase levels and extent of muscle damage in electrical burn, Burns, 30(7), 680-3.
5. Chemus C. (2014) Excizia grefare precoce, o metoda de tratament a mareluiars, Teza de doctorat, U.M.F. Carol Davila, Bucuresti.

6. Chiotan N. (1981). Un concept cibernetic al socului. Timisoara: Ed. Facla.

7. Isac Fl., Isac A., Bratu T., Tache M. \& Draghici I. (1994). Arsurile, Timisoara: Ed. Dinamis Print S.R.L. 